\title{
Smart Enterprise for Pulp \& Paper Mills: Data Processing and Reconciliation
}

\author{
M. Sánchez ${ }^{1}$, D. Leung ${ }^{2}$, H. Konecsny ${ }^{2}$, C. Bigaran ${ }^{2}$, J.A. Romagnoli ${ }^{3 *}$ \\ ${ }^{1}$ PLAPIQUI (UNS-CONICET), C. C. 717, 8000 - Bahía Blanca, ARGENTINA \\ ${ }^{2}$ Visy Pulp \& Paper Pty. Ltd. Tumut, NSW 2720, AUSTRALIA \\ ${ }^{3}$ Chemical Engineering Department, University of Sydney, Sydney, NSW, 2006
}

\begin{abstract}
An ad-hoc data reconciliation procedure developed for the recausticizing section of a new pulp and paper industry is presented in this work. A comprehensive model was formulated to take into account different unit operation modes. It was also extended to incorporate specific knowledge of some pieces of equipment to increase redundancy, and consequently enhance estimate precision and gross error detectability.
\end{abstract}

\section{Introduction}

Visy Industries, one of a few major players in the Australian paper business, has recently started up a state of the art modern pulp and paper making facility. The mill produces 240000 tonnes of Kkraft pulp and brown packaging paper each year; 800000 tonnes per annum of raw materials is sourced from local softwood plantations, that are supplemented by 50000 tonnes per annum of waste paper from domestic and commercial sources.

Smart Enterprise refers to a division at Visy Pulp and Paper Tumut that encompasses several areas of responsibility relating to data reconciliation, process modelling and simulation, process control and optimisation. Smart Enterprise's goal is to improve plant performance by allowing operators to make more informed decisions in a shorter amount of time.

With this objective in mind, data processing and reconciliation arises as a key component of Smart Enterprise, as raw data are processed by this technique to produce a consistent set of data that constitutes a reliable input for other procedures. Furthermore, it will also allow the detection of faulty sensors, bias in measurements or other anomalies in the process operating data. A comprehensive analysis of data reconciliation strategies can be found in Romagnoli and Sánchez ( 1999).

In this work we present the distinctive features of the data reconciliation procedure especially developed for the recausticizing section of the plant. To our knowledge, it is the first strategy reported for a pulp and paper industry. The methodology succeeds in considering changes in operating conditions associated with global plant production strategies or simply with equipment changes for maintenance cleanings. Furthermore extra knowledge is incorporated in the process model, by using pump characteristic curves, to overcome the lack of redundancy of the system. The procedure is

* to whom correspondence should be addressed 
complemented with a gross error detection technique based on the individual residuals test and a graphical interface in which input may be entered and results may be displayed. The graphical interface was generated using Excel with the data imported/exported directly between the two programs.

\section{Procedure Development}

Data Reconciliation is the process of adjusting or reconciling the process measurements to obtain more accurate estimates of flow rates, temperatures, compositions, etc... that are consistent with the material and energy balances. The classical procedure is based on the minimisation of the sum of the weighted difference between measured and estimated variables subject to a set of process constraints. It is valid under the assumption that no gross errors are present in the set of measurements. If it is not the case, gross error identification strategies are initially applied to determine and eliminate the set of suspect measurements.

Data Reconciliation strategies are supported by the location of a set of sensors in the plant, that allows the existence of redundant measurements. To know what type of information is available for a given process and set of instruments, an instrumentation analysis is performed. It allows classifying variables according to their feasibility of calculation. Measurements can be classified into redundant and non-redundant variables. The redundant ones are those whose value can be computed from the mathematical model that represents the plant and other measured variables. In turn, the unmeasured variables are called observable when they can be evaluated from the available measurements using model equations. Otherwise they are unobservable variables.

A data reconciliation procedure has been developed, following the classical approach, for the recausticizing area of the mill. This section plays two important roles in the production of white liquor. Firstly, it removes process impurities from the system in the green liquor filter. Secondly, the causticizing area increases the hydroxide (an important cooking chemical in the Kraft delignification process) content of the cooking liquor before it is fed into the digester area. The main features and motivations for each development stage of the procedure are presented below.

\subsection{Model formulation}

The model of the process is incorporated into the optimization problem as the set of constraints that should be satisfied at the solution point. But for the plant section under analysis, the model structure changes with time because the flow of some streams can be zero depending on the positions of 43 switches operated through the Distributed Control System. These streams are related to the green liquor feed (controlled from the recovery boiler) and to the cleaning cycles of the filters. Furthermore the presence of manual valves and streams connected to water locks can modify the model.

To tackle the problem of model variability with time, the broadest possible incidence matrix is first formulated. It has 146 rows and 306 variables associated with units and streams respectively. The model contains all streams interconnecting units (with the exception of those streams utilised only during plant maintenance) and fictitious streams 
that account for the net accumulation in the filters and tanks. It is assumed that only these units have an unsteady state operation mode.

A particular operation state is associated with specific positions for the switches, valves, and tank heights. Starting from this information and the all-inclusive model, a procedure was developed that determine the set of units and streams that participate in an operation state. It is based on row and column elimination of the general incidence matrix.

\subsection{Instrumentation Analysis}

Considering only mass balance calculations, an instrumentation analysis is performed to know what information is available from the process with the existing instrumentation, that consists of 17 magnetic flowmeters and 15 level sensors. The analysis is based on the variable classification procedure that uses the Q-R matrix decomposition (Sánchez and Romagnoli, 1996).

Different case studies are conducted whose results are included in Table 1. It contains the number of units and streams involved in the corresponding model, and the amount of redundant $(\mathrm{R})$, non-redundant (NR), observable $(\mathrm{O})$ and unobservable (UO) variables.

Normal operation condition is first considered (Case 1), i.e. all switch positions, height of tanks and valves are as indicated by the P\&I diagram as normal. In this case the number of measurements whose values can be adjusted through reconciliation, i.e. the redundant measurements, is zero. This is a serious drawback not only to enhance measurement's precision but to detect and identify gross errors.

Then a model that excludes vent tanks from the previous one is analysed (Case 2). Other vapour streams are also eliminated in Case 3, but the increment in system redundancy was not important. The incorporation of temperature measurements and energy balances in the model was discarded beforehand because its benefit was obviously worthless, due to the low number of temperature measurements that are present.

As any implementation of a data reconciliation procedure is settled on the existence of redundant variables, the next goal was to incorporate extra knowledge to the process model to increase system redundancy.

\begin{tabular}{ccccccc}
\hline \multicolumn{7}{l}{ Table 1: Instrumentation Analysis Results } \\
\hline Case & Units & Streams & R & NR & O & UO \\
1 & 120 & 262 & 0 & 32 & 31 & 199 \\
2 & 120 & 255 & 3 & 29 & 35 & 188 \\
3 & 106 & 218 & 3 & 29 & 46 & 140 \\
\hline
\end{tabular}

\subsection{Extended Model Formulation}

A study was undertaken to determine if measured variables related to pump operation could be used to formulate equations for flowrate adjustment, that is, relationships containing measured flowrates, constants and other measurements.

In the recausticizing area there are five centrifugal pumps of measured variable-speed. They are important pieces of equipment because they act as final elements of flowrate control loops that manage the input flow to filters and the slaker from tanks. The sensor configuration around each pump is shown in Figure 1. It consists of a magnetic 
flowmeter and two pressure gages installed on the pump output stream and a level sensor located on the tank, but there is no pressure sensors at pump inlet.

First a correlation was obtained using information from pump characteristic curves. It allows estimating the flow rate of liquor/slurry being processed by the pump in terms of the head and speed of the pump. This correlation is not useful for flowrate adjustment by itself because, the lack of pressure sensors at the pump suction avoids measuring directly the pressure rise of the liquid over the pump. Instead the energy balance of the fluid, from the liquid maximum height in the tank to the pressure sensor downstream of the pump, is used as an extra model equation because it contains all measured variables and constants. It is formulated as follows

$$
\frac{\mathrm{P}_{1}}{\rho \mathrm{g}}+\alpha_{1} \frac{\mathrm{v}_{1}^{2}}{2 \mathrm{~g}}+\mathrm{h}_{1}+\frac{\mathrm{W}_{\mathrm{s}}}{\mathrm{g}}+\mathrm{h}_{\mathrm{f}}+\mathrm{h}_{\mathrm{c}}=\frac{\mathrm{P}_{2}}{\rho \mathrm{g}}+\alpha_{2} \frac{\mathrm{v}_{2}^{2}}{2 \mathrm{~g}}+\mathrm{h}_{2}
$$

Where: $\mathrm{P}=$ Pressure $(\mathrm{kPa}), \rho=$ Density $(\mathrm{kg} / \mathrm{m} 3), \alpha=$ Velocity profile factor, $\mathrm{g}=$ Acceleration due to gravity $\left(\mathrm{m} / \mathrm{s}^{2}\right), \mathrm{h}=$ Height $(\mathrm{m}), \mathrm{W}_{\mathrm{s}} / \mathrm{g}=$ Shaft Work $(\mathrm{m}), \mathrm{h}_{\mathrm{f}}=$ Head loss due to friction $(\mathrm{m}), \mathrm{h}_{\mathrm{c}}=$ Head loss due to contractions $(\mathrm{m})$.

In equation (1), the velocity in the tank is assumed to be zero, the pressure at point 1 is given by the tank pressure (usually atmospheric), the pressure at point 2 is read from the pressure indicator, the height terms are derived from the level indicator on the tank and the geometry of the pipe work obtained from isometric drawings, the head loss due to friction and contractions is formulated in terms of pipe geometry and flow rate.

The process model is enlarged with seven non-linear semi-empirical equations, corresponding to balances indicated by formula (1). Also redundant equations that consider the equality of pressure due to hardware redundancy are included in the model. For the normal operation state, the results of variable classification are: $R=25, N R=22$, $\mathrm{O}=32$, $\mathrm{UO}=197$. Redundant measurements include flowrates, but also pressures and pump speeds that has not been considered in Case 1.

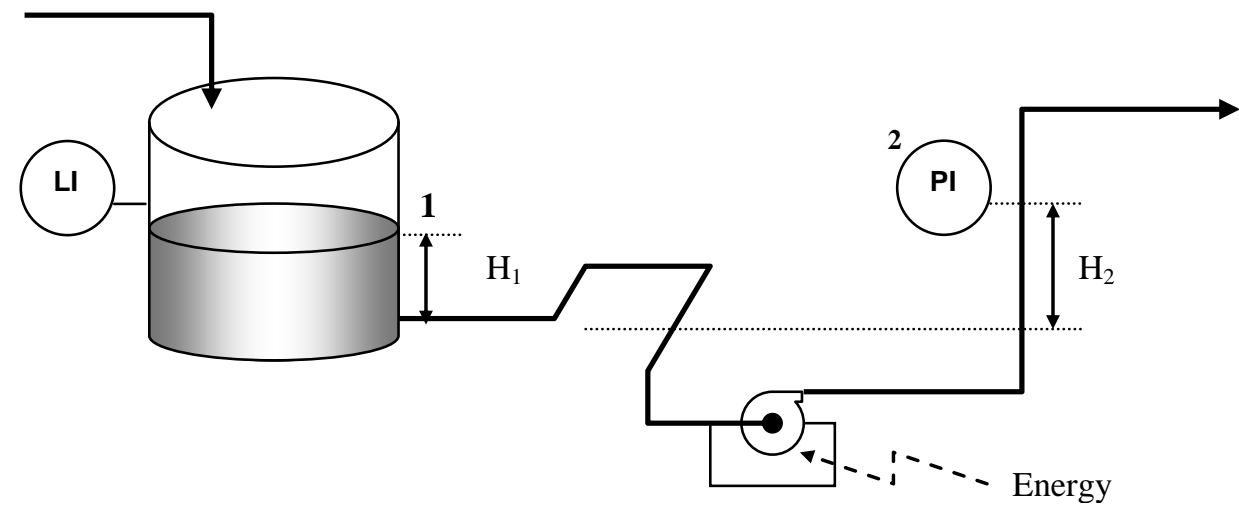

Figure 1: Tank and Pump Configuration

\subsection{Data Reconciliation}

A current set of measurements and switch positions is provided by the DCS at intervals of $2.5 \mathrm{~s}$. The current operating status is compared with the previous one. If the 
operational mode had changed the model us modified accordingly and the DR is performed on the measured redundant variables. Its inputs are the vector of average measurements, obtained from sensor outputs corresponding to the same operational state, and the current sector model is made up of the mass balances associated to nonzero flow streams and the redundant equations obtained for pump operation. If there is no change in the operational state of the process, the DR procedure is performed after a prefixed interval of ten minutes. Two data reconciliation procedures were derived, linear and non-linear. The linear one, uses the linearized version of the redundant model, consequently estimates are calculated straightforward. The solution of the nonlinear problem was achieved using a SQP technique. Measurement errors are estimated using the information provided by the vendors. A graphical interface was developed in Excel to allow the model's results to be presented in a recognizable and easy to read format. Figure 2 below shows a sample of the on-line results for reconciled and measured values in the case of the dregs filter pressures.
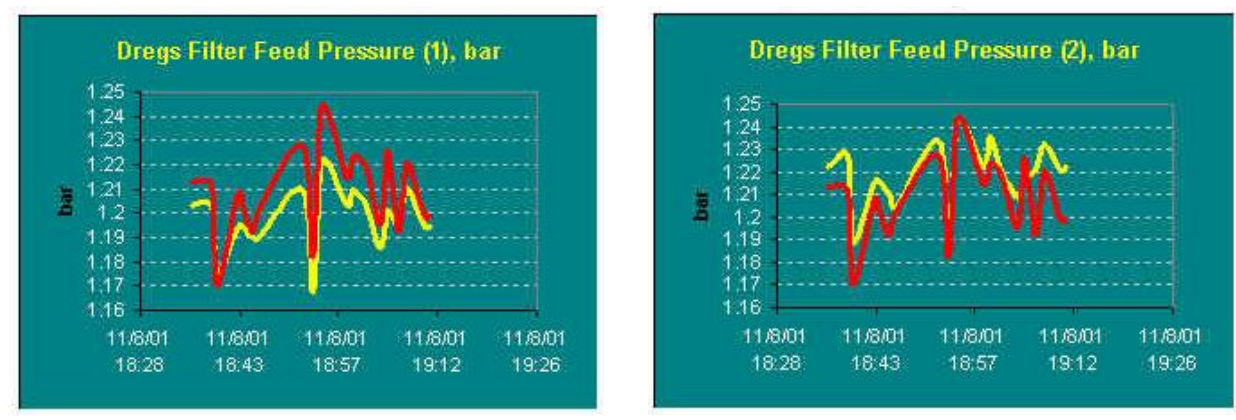

Figure 2: DR results for dregs filter pressures

\subsection{Gross Error Detection}

Data reconciliation deals with the problem of random errors. If gross errors are also present in the process data, they must be identified and removed before reconciliation. In our approach we have used the test on the residuals for gross error identification. Following Mah and Tamhane (1982) and defining the residuals as

It is easy to show that

$$
e=y-\hat{y}
$$

$$
e \sim \mathrm{N}(0, V)
$$

Where, $\mathrm{V}$ is the covariance of the residuals. Therefore, if there is no gross error in the ith observation

$$
e_{i} \sim \mathrm{N}\left(0, v_{i i}\right)
$$

We conclude that there is a gross error present in the ith observation, if

$$
\left|E_{i}\right|=\left|e_{i}\right|>E_{\alpha / 2}
$$


Where $E_{\alpha / 2}$ is the upper $\alpha / 2$ point of the standard normal distribution and $\alpha$ is the level of significance. In our case, $E_{\alpha / 2}$ was obtained from the actual distribution of the plant data by approximating the distribution function of the residuals, based on historical information for normal operating conditions. Figure 3 illustrates the bar plot of the residuals (critical value based on statistics $\left(E_{\alpha / 2}\right)$ and actual value $e_{i}$ after reconciliation) for two operating conditions.

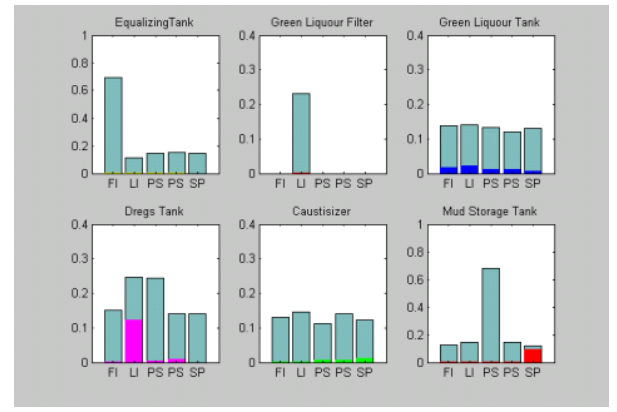

(a)

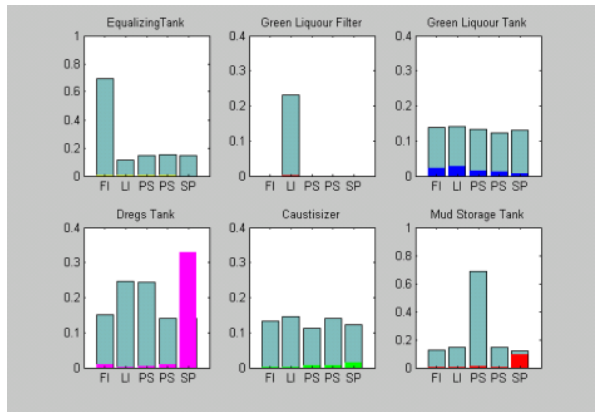

(b)

Figure 3:Residuals plot (a) Normal condition (b) Faulty condition

\section{Conclusions}

In this work, the study conducted for implementing a data reconciliation procedure at the recausticizing sector of the Visy Pulp and Paper Mill, Tumut is presented. The procedure points towards solving two main aspects. One of them is the variability with time of the model representing sector operation, a point not considered in previous data reconciliation procedures that work for only one operative mode. The second aspect involves the necessity of increasing system redundancy. In this sense semi-empirical relationships among measured variables are obtained using information from pump characteristic curves. This constitutes also an innovative way to overcome the difficulties associated with the lack of instrumentation.

\section{References}

Romagnoli, J.A. and M. Sánchez, 1999, Data Processing and Reconciliation for Chemical Process Operations. Academic Press, San Diego.

Sánchez, M. C. and J. Romagnoli, 1996, Use of Orthogonal Transformations in Classification/Data Reconciliation. Computers and Chemical Engineering, 20, 483- 493.

Mah R.S.H., Tamhane A.C., Detection of gross erros in Process Data, AIChE Hournal, Vol. 28, No. 5 pp. 828-830 (1982). 